\title{
Universität Berlin
}

Medizinische Behandlungen mit Eigenblut (überwiegend venose Entnahme und intramuskuläre Reinjektion) sind typi-sche Methoden einer «umstimmenden» und konstitutionver-bessernden Therapie. Derart verwendetes Blut bedeutet einen «Reiz», welcher gesundende Reaktionen des Organismus anregen soil.

Es gibt verschiedene Versuche, dieses Blut vor der Reinjektion zu modifizieren, es dem Organismus gewissermassen «fremd» zu machen; damit soil der therapeutische Reiz solcher Behandlungen verstärkt werden. Die bekannteste Methode ist heute eine vorherige Bestrahlung des Blutes mit ultraviolet-tern Licht, üblicherweise werden etwa $50 \mathrm{ml}$ Blut entnommen und venös zurückgegeben (sog. UVB). Bei der Hämatogenen Oxidationstherapie (HOT) wird das Blut vor der UV-Exposi-tion mit Sauerstoff aufgeschäumt.

In der võrantibiotischen Ära fand diese Behandlung eine um-fangreiche Anwendung vor allem bei Infektionserkrankungen. Später wurden dann auch angiologische Indikationen ent-wickelt. Weitere, häufig behandelte Erkrankungen stammen aus dem dermatologisch/allergologischen, metabolischen (auch Fettleber) und onkologischen Formenkreis. Nachdem diese

Behandlungsmethode bis in die 50er Jahre auch in angesehenen Kliniken angewendet wurde und auch in seriösen «schulmedizinischen» Zeitschriften dargestellt worden ist, gilt sie heute als Methode der Aussenseiter. Eine relevante wissenschaftliche Bearbeitung fand zuletzt nur noch in einigen «sozialistischen» Ländern (insbesondere der DDR) und vor allem in der Sowjetunion statt. Allerdings wurden die russischen Arbeiten im Westen kaum rezipiert. Das von der Fa. Eumatron/München gesponserte Symposion sollte einer wissenschaftlichen Bestandsaufnahme dienen, vor allem soil-ten die russischen Ergebnisse bekanntgemacht werden.

Grundlagenforschungen von R. Dehmlow, Berlin, hatten sich mit Veränderungen von Eiweissfraktionen im Blut unter einer UVB/HOT beschäftigt. Er konnte nachweisen, dass aus mono-merem Humanserumalbumin (HSA) Dentamere (1Ofaches Molekulargewicht mit etwa 690 kD) gebildet werden. Das aggregierte Protein konnte isoliert und charakterisiert werden, seine physikalisch-chemischen und biologischen Eigenschaften wurden bis zu den therapeutischen Wirkungen analysiert. Diese neuen HSA-Moleküle lösen nach Reinfusion des be-strahlten Blutes über Entzündungsmediatoren kurzzeitig Ent-zündungsprozesse und eine Aktivierung des Immunsystems aus. Die Mediatoren, z. B. Histamin, Prostaglandine, Interleukine usw. entstehen bei der Degranulation der basophilen und neutrophilen Granulozyten. Die aggregierten Moleküle wirken entsprechend als Immunmodulatoren. Durch diese ausgelösten Prozesse sind auch die sogenannten «Erstverschlimmerung» und der oftmals auftretende «Flush» unter der Therapie erklärbar.

G. und U. Frick, Greifswald, stellten Ergebnisse eigener hämo-staseologischer Untersuchungen vor. Danach kommt es unter einer UVB-Bestrahlung zu einer Vermehrung von Heparino-zyten und zur Freisetzung saurer Mucopolysaccharide vom Heparin-Typ, zu einer 
Fibrinogenverminderung und Änderun-gen der Fibrinogenstruktur und -reaktivität, zur Aktivierung der Fibrinolyse, zu einer Senkung der Thrombophilie bei Herz-KreislaufErkrankungen (Verminderung von Hyperlipidämie und Plasmaviskosität) sowie zu einer initialen Thrombozyten-aggregation mit nachfolgender Langzeitnormalisierung der Thrombozytenfunktion. Bei gleichzeitigen günstigen Wirkungen auf hämorheologische Eigenschaften des Blutes kann langfristig mit einer verminderten Atherogenese gerechnet werden.

G. Lewin und I. Popov, Berlin, interpretierten UVB bzw. HOT als einen pseudopathologischen prooxidativen Reiz, der unter der Voraussetzung einer optimalen Dosierung eine Gegen-reaktion im Organismus stimuliert. In einer Therapiestudie bei Patienten mit Claudicatio intermittens war das günstige kli-nische Resultat mit einer Verbesserung der antioxidativen Kapazität des Blutplasmas korreliert. In einer anderen Patien-tengruppe mit eher negativen Wirkungen auf das psoriatische Krankheitsbild war gleichzeitig diese antioxidative Kapazität verschlechtert worden. Insofern sei UVB/HOT mit externer Heliotherapie vergleichbar, sie ermögliche aber eine sorgfälti-gere Dosierung der Reizintensität.

A. Turowski und M. Paulitschke, Berlin, berichteten über eine unkontrollierte

Anwendungsbeobachtung bei 19 Patienten mit chronischen Lebererkrankungen unterschiedlicher Ätiologie, welche mit einer HOT-Serie behandelt worden waren. Bei Patienten mit Leberzirrhose und chronischer Hepatitis sahen sie eine Verbesserung der subjektiven Symptomatik (Müdig-keit und Konzentrationsstörungen), Verbesserungen der Syn-theseleistung der Leber erkannten sie an einem signifikanten Anstieg des Prothrombinwertes und plasmatischer Eiweiss-spiegel. Bei pathologischen Ausgangswerten nahmen plasma-tische Transaminasen ab.

Der Beitrag Samoilowa fasste die klinischen Arbeiten im russischen Sprachraum und eigene Grundlagenforschung zu-sammen. Danach werden entsprechende Geräte in jeder bedeutenderen Klinik umfangreich eingesetzt. Eine selbstent-wickelte Apparatur arbeitet mit einer Strahlendosis von etwa $150 \mathrm{~J} / \mathrm{m} 2$. Durchschnittlich werden 2-5 Behandlungen in einem Abstand von 1-2 Tagen durchgeführt. Jedesmal werden

(C) 1995 S. Karger GmbH,

Freiburg

1-2 ml Blut pro kg Körpergewicht behandelt. Die Indika-tionen sind mit den unsrigen in etwa vergleichbar. In der Notfallmedizin wird die Behandlung bei akuten Infektionen, Sepsis, Peritonitis, Traumata und Verbrennungen eingesetzt. Umfangreiche eigene Grundlagenforschung in dem von Frau Samoilowa geleiteten Zytologischen Institut der Akademie der Wissenschaften in St. Petersburg hat sich mit strukturellen und funktionellen Eigenschaften der roten und sämtlicher weisser Blutzellen sowie der Thrombozyten und mit Repair-Mechanismen der DNS nach artifiziellen Schädigungen be-schäftigt. Ähnliche Fragen wurden auch für Bindegewebszellen und Zellen des Knochenmarkes gestellt. Regelmässig wurden Wirkungen der UVExposition selbst untersucht (bestrahltes Blut) sowie Wirkungen, wenn das UV-bestrahlte Blut rückinjiziert worden war.

Zum Beispiel beobachtete Frau Samoilowa eine sehr ausgeprägte Stimulierung der phagozytären Aktivität von Granulozyten, allerdings nur, wenn in dem bestrahlten und rückinjizierten Blut gleichzeitig Monozyten und Lympho-zyten anwesend gewesen sind. Ähnlich war eine Stimulierung der DNS-Synthese in Lymphozyten von der Anwesenheit von Monozyten in bestrahltem Blut abhängig. Die syste-mische Wirkung UV-bestrahlten Blutes nach Rückinjektion war wesentlich ausgeprägter als die Wirkung der Bestrahlung selbst (Messungen nur in dem UVexponierten Blut). Auch die phagozytäre Aktivität von Monozyten wird signifi-kant angeregt. 
Dabei sind die Wirkungen starker, wenn bestrahltes mit nicht bestrahltem Blut gemischt wird, als in dem bestrahlten Blut selbst. Zu dieser vermehrten Phagozytose kam es nur, wenn in dem bestrahlten Blut auch mononukleäre Leukozyten anwesend waren. Durch eine Bestrahlung der übrigen Zellen und des Blutplasmas konnten entsprechende Effekte nicht hervorgerufen werden. Gleichzeitig kommt es unter der Bestrahlung wie auch unter der Wirkung bestrahlten Blutes zu einer Sekretion verschie-dener Zytokine wie Interleukin 1 und Tumornekrosefaktor, die vielfältigen Wirkungen dieser Substanzen sind gut be-kannt.

In Versuchen mit menschlichen Krebszellen konnte gezeigt werden, dass die Aktivität natürlicher Killer-Zellen im Laufe von 30-60 min nach der Reinfusion bestrahlten Blutes ansteigt.

Die Behandlung mit UV-bestrahltem Eigenblut fördert auch die Wundheilung und die Abheilung gastrointestinaler Ge-schwüre sowie Ulcera der Haut. Vor diesem Hintergrund interessierte auch, dass das Wachstum menschlicher Fibro-blasten unter dem Einfluss bestrahlten Blutes signifikant stimuliert wird (In-vitro-Versuche mit embryonalen Zellen). Die Ergebnisse deuten auf eine Freisetzung entsprechender Substanzen aus Monozyten, Lymphozyten und Thrombozyten hin. Grundsätzlich ähnliche Beobachtungen wurden auch in Zellkulturen mit Knochenmarkszellen gemacht. Bei vergleich-baren Experimenten mit malignem Zellgewebe (Hyper-nephrom) kam es zu keinem vermehrten Wachstum. In Kulturen mit experimentell gestörter DNS konnten RepairProzesse durch UV-bestrahltes Blut angeregt werden, im weiteren Verlauf wurden weniger Chromosomenaberrationen beobachtet.

Umfangreiche Untersuchungen zu Membranstrukturen und funktionellen Veränderungen in diesem Bereich sollen hier nicht mehr referiert werden. Die bekannten Verbesserungen der Hämorheologie fanden ihr morphologisches Substrat z. B. in einer verbesserten Verformbarkeit und geringeren Aggregation von Erythrozyten.

Die Autorin stellte immer wieder heraus, dass vor allem pa-thologisch veränderte Funktionen verbessert wurden. Es kann also mit einer gewissen Normalisierung durch eine solche Therapie gerechnet werden. Ausgeprägte Reaktionen seien vor allem in der sonnenarmen Jahreszeit beobachtet worden, während des Hochsommers - mit ausreichend natürlicher UV-Bestrahlung komme es zu entsprechenden Veränderungen nicht bzw. kaum.

In der abschliessenden Diskussion wurden Behandlungen mit UV-bestrahltem Eigenblut als eine wissenschaftlich hochinteressante, klinisch erfolgversprechende Methode eingestuft. Zumindest im westlichen Sprachraum fehlen kontrollierte Therapiestudien nach den heute geforderten wissenschaftlichen Protokollen. Zur Grundlagenforschung erscheinen jetzt vor allem immunologische Fragestellungen als bedeutsam. Entsprechende Ergebnisse konnten wahrscheinlich auch zum Verständnis einer Wirksamkeit bei angiologischen Patienten nützlich sein. M. Bühríng, Berlin

R. Dehmlow, Berlin

220

B ühring/D ehmlow

Kongressbericht - Congress Report 